\title{
Negative Effects of COVID-19 Stay-at-Home Mandates on Physical Intervention Outcomes: A Preliminary Study
}

\author{
John Michael Templeton*, Christian Poellabauer and Sandra Schneider \\ Department of Computer Science and Engineering, University of Notre Dame, Notre Dame, IN, USA
}

Accepted 23 March 2021

Pre-press 13 April 2021

\begin{abstract}
.
Background: Due to the COVID-19 pandemic, beneficial physical intervention classes for individuals with Parkinson's disease (PD) were cancelled.

Objective: To understand effects of the COVID-19 stay-at-home mandate and the inability to participate in recommended and structured physical interventions as a consequence of these mandates, specifically designed mobile assessments were used that collected both self-reporting information and objective task-based metrics of neurocognitive functions to assess symptom changes for individuals with PD.

Methods: Self-reporting questionnaires focusing on overall quality of life (e.g., when individuals typically feel at their best, changes in activity levels, and symptom progression) were given to all individuals $(n=28)$. In addition, mobile-based neurocognitive assessments were administered to a subset of the population $(n=8)$ to quantitatively assess changes due to COVID-19 restrictions.

Results: The highest self-reported factors in which individuals denoted feeling their best were after exercise (67.86\%) and being in a comfortable and supportive environment $(60.71 \%)$. Objective measures found overall duration of physical activity during the stay-at-home mandate decreased significantly $(p=0.022)$. With the lack of overall activity, $82.14 \%$ of individuals self-reported having at least one symptom that worsened moderately or higher. Further testing, using mobile-based assessments, showed average completion times of functional tasks increased, taking about 2.1 times longer, while accuracy metrics showed overall degradation.

Conclusion: Although the COVID-19 stay-at-home mandate was intended to help protect individuals at high risk from coming into contact with the virus, it also prevented individuals from receiving recommended supervised exercise interventions resulting in significant negative effects in social well-being and across motor and speech neurocognitive tasks for individuals with PD.
\end{abstract}

Keywords: COVID-19, Parkinson's disease, physical interventions, neurocognitive assessment, quality of life

\footnotetext{
*Correspondence to: John Michael Templeton, MSc, Department of Computer Science and Engineering, University of Notre Dame, 384 Fitzpatrick Hall, Notre Dame, IN 46556-5637, USA. Tel.: +1 480296 8562; E-mail: jtemplet@nd.edu.
}

\section{INTRODUCTION}

As individuals age there is the progressive decline of many physiological functions and an increased susceptibility to certain diseases [1]. The neurodegenerative association between aging and Parkinson's 
disease (PD) has been recognized for several decades [2]. Neurodegenerative diseases present with progressive degeneration of neurons and neural structures and this degeneration in PD can ultimately lead to both movement disorders and neurological and/or cognitive disorders [3]. Neurological disorders in PD manifest when regions in the brain that play a key role in movement are affected, which may lead to symptoms such as tremors, muscle stiffness, impaired speech production, lack of facial expressions, and freezing of gait [4-6]. Cognitive disorders occur when regions responsible for mental functions, including memory, judgement, planning, and behavior, undergo degeneration [7, 8]. Combinations of these motor and cognitive disorders can have dependencies on the individual, their age, gender, socioeconomic background, as well as the stage, severity, and progression of the disease [9]. Any or all of the aforementioned symptoms, experienced by these individuals, can significantly deteriorate their overall quality of life.

PD is often described as a "designer disease", meaning no two people diagnosed manifest the exact same symptoms [10]. Frequently, the definitive diagnoses of PD usually come after a prolonged period of time due to vague, initial symptoms [11]. By the time some individuals are diagnosed with PD they likely have already experienced limitations in activities of daily living and thus are in need of immediate rehabilitation [11]. The recommendations of both pharmacological and therapeutic treatments are intended to combat the continued progression of the disease. While there are now many different therapy options available for diagnosed populations, physical activity is the most effective non-pharmacological aid to individuals with PD [4]. Even in the case of pharmacological interventions, physical activity is increasingly advocated as an adjunct intervention [12]. The majority of these physical rehabilitation activities are conducted outside the home in supervised settings (e.g., non-contact boxing programs, supervised aerobic or functional strength activities, and yoga) even though many activities are suitable for at home completion [11]. Programs, that can include flexibility, balance, functional movement and strength, and aerobic exercise, can be individually tailored and/or administered in group settings to help individuals with PD engage in beneficial physical activities [11, 13].

With the ongoing COVID-19 pandemic, health authorities (e.g., the Centers for Disease Control and Prevention) warned older populations that they were at a higher risk of more serious and possible fatal illness associated with COVID-19 [14]. The global recommendation for these older populations included social isolation (e.g., staying at home and avoiding social contact with family members and friends, for extended periods of time). However, these recommendations can have serious consequences for all older individuals, but particularly for individuals with $\mathrm{PD}$, who are at risk for increased disease progression due to limited physical, social, and mental activities. The objective of this paper is to address the impacts of the COVID-19 stay-at-home mandates on individuals with PD due to the restrictions enforced by the pandemic. An understanding of individuals' symptom progression, due to the COVID-19 stay-athome mandate, is of interest. Symptom progression should be obtained in the form of both self-reported symptoms and objective measures from neurocognitive assessments. This paper focuses on self-reported physical activity levels of individuals with PD as well as qualitative changes in their neurocognitive symptoms. Further focus is placed on the collection and analysis of quantitative metrics, gathered from neurocognitive assessments, to understand the accuracy of individuals' self-reported symptoms.

\section{Related work}

\section{Interventions}

Previous studies suggest that individuals with PD should interact with physical intervention activities as an adjunct intervention to pharmacological therapies $[4,12]$. Structured intervention programs also include natural and beneficial aspects of social engagement that group exercise programs can provide $[15,16]$. Recent social engagements for individuals with PD are also associated with better global cognition, whereas low levels of social engagement were associated with an increased risk of dementia [17]. Further, interventional programs that enhance social support for older people may decrease mortality and produce public health benefits [18]. Due to the COVID19 pandemic, and subsequent stay-at-home orders, beneficial physical intervention classes and social engagement opportunities for individuals with PD were cancelled due to the higher risk associated with COVID-19 [14]. Public health emergencies, such as COVID-19, can also affect the well-being of individuals (e.g., emotional isolation and deficient medical resources). Further, groups such as the elderly and people with preexisting medical conditions like PD may be at increased risk for adverse psychosocial 
outcomes including stress, depression, insomnia, fear, and confusion $[16,19]$.

\section{Self-reporting}

Self-reporting is a common way to monitor shortterm (e.g., day to day) changes, but can also lead to improved disease management by allowing the individual to recognize and understand their condition and to be aware of their symptoms and triggers [20]. Exploratory analyses show that high self-reported physical activity is associated with less disease progression of individuals with PD and suggests that especially in the early stages of $\mathrm{PD}$, could potentially have a positive impact on the disease course [21].

\section{Functional assessments}

Self-reporting should be supplemented by objective measures (e.g., functional assessments) to further understand individuals' symptoms, neurocognitive capabilities, and the individuals' awareness and accuracy of the disease progression. These self-reporting and functional assessments should occur across all functional areas of neurocognition (e.g., motor, memory, speech, language, executive function, sensory, behavioral and psychological state, sleep, and autonomic function [9]. While there are many screening (e.g., Montreal Cognitive Assessment, Mini-Mental State Examination, and the Menu Task Assessment) and comprehensive assessments (e.g., Boston Diagnostic Aphasia Examination, Dean-Woodcock Neuropsychological Assessment System and Neurobehavioral Functioning Inventory), mobile-based neurocognitive assessments, utilizing the same functional tasks, provide many benefits [22-27]. Mobile versions of functional assessments can be highly beneficial for both users and clinicians in the reduction of subjective biases due to the collection of objective measures. The collection of objective metrics, using mobile devices, were utilized to confirm self-reported symptoms, and are intended to aid in the formation of enhanced, objective datasets [9].

\section{METHODS}

\section{Self-reporting}

To minimize interpersonal interactions due to COVID-19 mandates, questionnaires were utilized to gain an understanding of individuals' quality of life. Questions included the times of day in which individuals typically feel at their best, their activity levels prior to their diagnosis, after their diagnosis, and after 4 months (Early March - Late July) of stay-athome mandates and in-person intervention program restrictions. Questions were also included that asked about the individual's symptoms and their subsequent progression before and after these restrictions. The questionnaire is included in Supplementary Table 1. Self-reporting questionnaires were collected from 28 individuals with PD between the ages of 52 and 84 . About half ( $n=15$ or $53.57 \%$ ) of the population was female, and the average disease duration across the population was 6.07 years. All reporting individuals participated regularly (e.g., at least twice a week) in structured in-person intervention programs led by certified fitness instructors prior to the COVID-19 stay-at-home mandates. The in-person intervention programs have been set up specifically for individuals with PD and they occur at local health and fitness centers with access to a variety of training and exercise equipment. A subset of these individuals also completed physical exercise either at home or outside these structured intervention programs. Individuals were recruited to participate in this IRB approved study via advertisement through the structured intervention programs, physician and clinician referrals, and prior studies from our laboratory. Given the mean age of onset for PD in the Western world is early-tomid $60 \mathrm{~s}$ [28], we limited our recruitment efforts to diagnosed individuals age 50 years or older. Participants were excluded from the current study if they were unable to provide written informed consent. All participants were local to the Midwest of the United States of America in both Indiana and Michigan and therefore were subject to the local mandates of those respective states and counties. All individuals were required to denote their activity levels by the number of active days per week, the average duration of activity per day, and the activities they complete. Further they were asked to self-report and evaluate changes across common PD symptoms (e.g., fatigue, impaired handwriting, quiet speech, tremor, forgetfulness, etc.) Changes in common PD symptoms were denoted on a Likert scale of 1-5, with 1 indicating no change in the symptom and 5 indicating very severe changes.

\section{Mobile application testing}

To gain a quantitative measurement of symptoms, a subset of the 28 diagnosed individuals $(n=8)$ completed a mobile-based neurocognitive assessment, which occurred approximately 5 months after the start of the stay-at-home mandate (e.g., August). A subset of the population was used as not all 
participating individuals were able to or had returned to in-person physical intervention classes following the stay-at-home mandates. This was due either to additional restrictions set by state departments, regarding the opening of various facilities, or individuals maintaining distancing protocols as they are notably at higher risk due to comorbidities. The objective metrics collected from this assessment tool were compared to their last screening, using the mobile-based neurocognitive assessment, which occurred prior to the COVID-19 stay-at-home mandate (late February to early March) as part of a regularly scheduled data collection. The collection of objective metrics, gathered from a designed, mobile-based neurocognitive assessment tool, was completed to understand the accuracy of individuals' self-reported symptoms. This assessment tool was specifically designed for individuals with PD that focused on user-device interactions for the collection of objective measures [29]. All functional assessments and selfreporting were either administered or input on the mobile device that provided the data. Functional tasks included in the mobile-based neurocognitive assessment included motor, memory, speech, and executive function tasks. All participants were required to complete mobile versions of functional tasks (e.g., tracing shapes, apraxia tests, reflex tasks, card matching, trail making, and speech-based assessments). For a finemotor tracing task the individual is instructed to use their index finger to trace a depicted shape, in a clockwise motion starting from the left. In a gross-motor task the user was to manipulate the mobile device to "air"-trace a prompted shape (e.g., a square). For reflex tasks, the user is intended to tap on the screen to interact with a set of targets. For a memory task the user is to tap on depicted cards until all cards have been matched in pairs. In a trail making task the user is intended to draw a line using their index finger to connect the shapes in increasing numerical order. For a set of speech-based tasks, the user is instructed to read a sentence out loud or name prompted objects. Finally, a set of tasks also implement dual-task interference for the understanding of how these individuals interact in multifunctional task approaches. Examples of dual-tasks include both fine (e.g., tracing an object) and gross (e.g., manipulating the mobile device) motor tasks paired with a nonautomatic speech task (e.g., listing the months of the year, aloud, in reverse order; December to January). Additionally, executive functional tasks are also dualtask by nature as the individual must "put into action" their necessary 'executive functions'. This is seen in the Stroop Word Color Test (SWCT) as the user is required to discern the difference between prompted colors and words and then speak the correct response.

\section{Standard protocol approvals}

This work presented in this manuscript is part of an IRB approved study. Written informed consent was collected from all participants included in this study. The collected data was authorized for disclosure as part of published works.

\section{Data availability statement}

Individual de-identified participant data will not be shared at this time. The data collected for this work is part of a larger dataset for concurrent projects on mobile based neurocognitive assessments for individuals with PD.

\section{RESULTS}

\section{Self-reporting}

\section{Quality of life}

Figures 1 and 2 show the responses from the self-reporting surveys for different factors in which diagnosed individuals with PD feel at their best. Individuals were able to report factors based on both the time of day and typical daily events or settings that contributed to them feeling at their best. Categories based on the time of day include: early morning to late morning (EM-LM), late morning to midday (LM-MD), midday to mid afternoon (MD-MA), mid afternoon to evening (MA-EV), and evening to before bed (EV-BB), as seen in Fig. 1.

Additional factors are seen in Fig. 2, which include after eating (AE), after taking their prescribed medications (AM), after physical activity or exercise (AEx), and being in a comfortable environment (CE). Both timing and additional factors were included to gain valuable insights on the individuals' quality of life. In Fig. 1, the highest reported instances overall show individuals feeling at their best earlier in the day, then trending down in the evening. However, the highest reported instances of individuals feeling at their best were after exercise and being in a comfortable environment (Fig. 2).

\section{Activity}

Table 1 shows the activity levels of individuals diagnosed with PD prior to their diagnosis (BD), after 


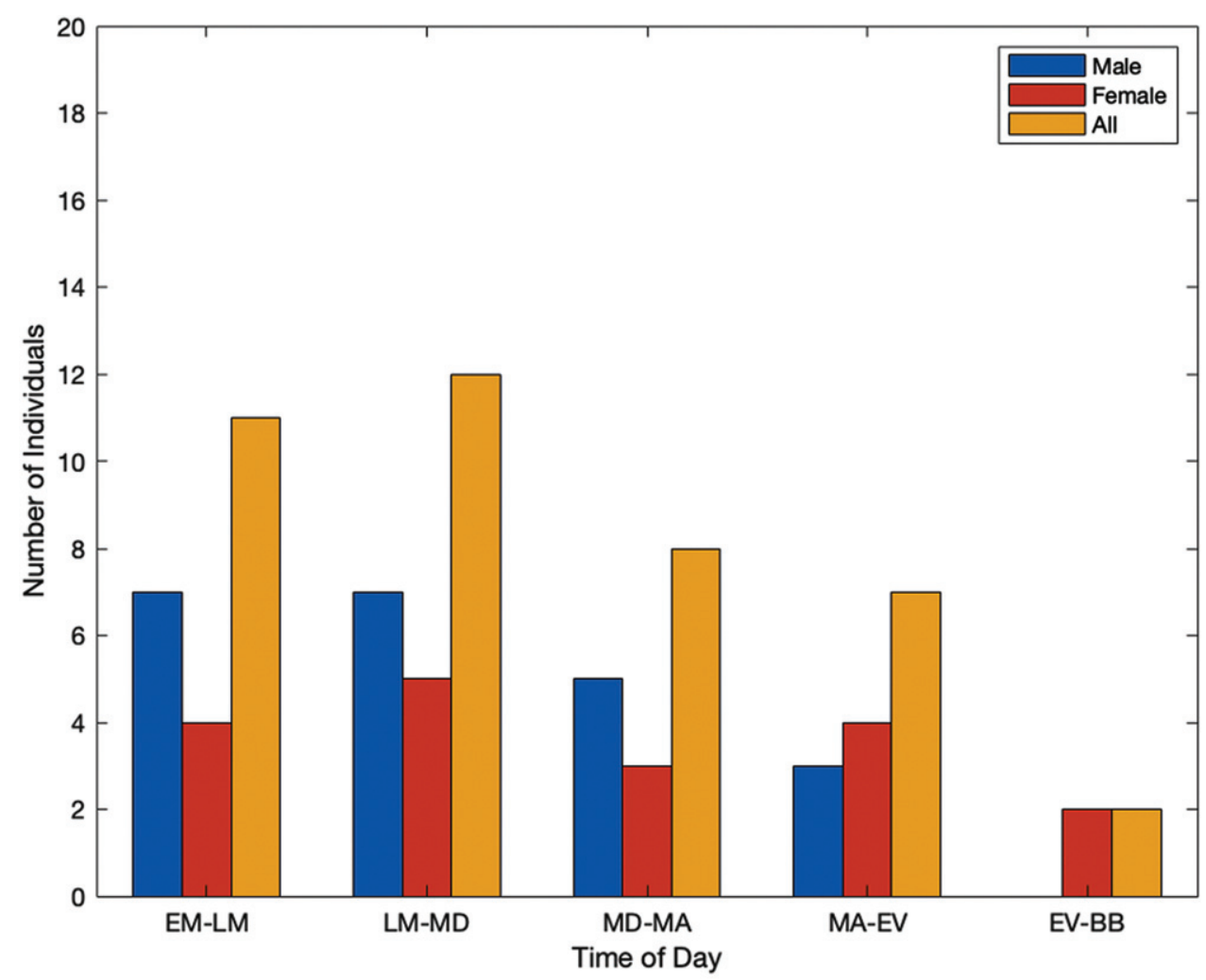

Fig. 1. Time of day in which individuals with PD feel at their best.

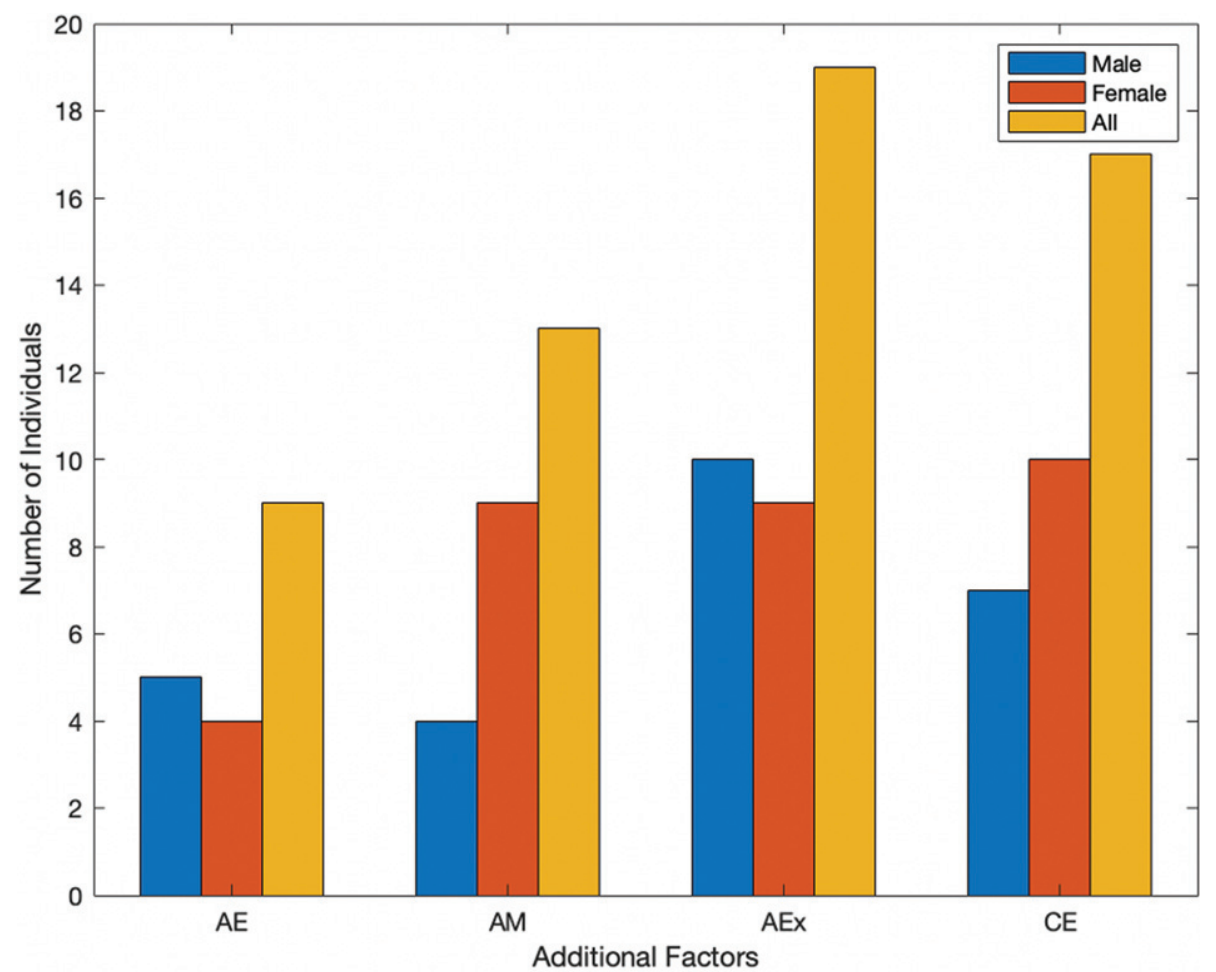

Fig. 2. Additional factors in which individuals with PD feel at their best. 
Table 1

Individuals' activity levels prior to their diagnosis, following their diagnosis, and during the COVID-19 stay-at-home mandate

\begin{tabular}{lccc}
\hline Metric & BD & AD & SaHM \\
\hline Number of Active Days & & & \\
$\quad$ Male & 4.2 & 4.62 & 4.62 \\
$\quad$ Female & 3.2 & 3.73 & 3.4 \\
$\quad$ All & 3.78 & 4.14 & 3.96 \\
Number of Active Minutes & & & \\
$\quad$ Male & 62.69 & 75.77 & 48.46 \\
Female & 37.86 & 43.21 & 30.71 \\
All & 49.81 & $\mathbf{5 8 . 8 9 ^ { * }}$ & $\mathbf{3 9 . 2 6}$ \\
Number of Activities & & & \\
$\quad$ Male & 11 & 14 & 8 \\
Female & 13 & 14 & 6 \\
All & 17 & 18 & 11 \\
\hline
\end{tabular}

$\mathrm{BD}$, before diagnosis with Parkinson's disease; $\mathrm{AD}$, after diagnosis with Parkinson's disease; SaHM, after the COVID-19 stay-athome mandate.

their diagnosis (AD), and after the 4-month stay-athome mandate and program restrictions put into place for COVID-19 (SaHM). Individuals reported minimal change in the number of active days per week; however, both the number of active minutes per day and the number of total activities showed a notable decrease due to the COVID-19 restrictions.

\section{Symptoms}

Table 2 shows changes in the symptoms of individuals diagnosed with PD since the stay-at-home mandate and program restrictions were put into place for COVID-19. The top portion of Table 2 depicts the number of individuals who had at least one symptom change moderately or higher, whereas the bottom portion shows the number of individuals who had multiple symptoms worsen moderately or severely. The lower portion of Table 2 is a subset of the upper responses.

A depiction of the extent individuals reported changes of symptoms across neurocognitive areas of

Table 2

Number of individuals with changes in symptom(s) since the stayat-home mandate based on a Likert Scale of 1-5. 'Moderate +' is indicated by a Likert response of 3 or higher. 'Severe + ' is indicated by a Likert response of 4 or higher

\begin{tabular}{lcc}
\hline Group & Moderate + $(\%)$ & Severe + $(\%)$ \\
\hline At Least One Symptom & & \\
Male & $10(76.92 \%)$ & $6(46.15 \%)$ \\
Female & $13(86.67 \%)$ & $10(66.67 \%)$ \\
All & $23(82.14 \%)$ & $16(57.14 \%)$ \\
Multiple Symptoms & & \\
Male & $9(69.23 \%)$ & $5(38.46 \%)$ \\
Female & $10(66.67 \%)$ & $7(46.67 \%)$ \\
All & $9(67.86 \%)$ & $12(42.86 \%)$ \\
\hline
\end{tabular}

interest are seen in Fig. 3. The reported symptoms for each group (e.g., male, female, and overall) were based on average self-reporting metrics from a Likert scale of 1-5, with 1 indicating no change in the symptom and 5 indicating very severe changes. The highest reported symptoms that worsened for the representative population included autonomic function, speech, and motor function. Women also indicated sleep changes as a largely and negatively impacted symptom. Figure 3 gives average self-reporting values of each group, for all functional categories of neurocognition. On average, all neurocognitive functions of interest worsened for the overall population as Likert values were all greater than 1 (Fig. 3).

\section{Mobile application testing}

Eleven functional tasks were completed on a mobile device for the collection of objective metrics across neurocognitive functional areas of interest. Tasks included tracing shapes, apraxia tasks, reflex tasks, card matching, trail making, object naming, and dual-tasks (e.g., completing two tasks simultaneously). The metrics considered for all functional tasks include both temporal and accuracy metrics. All metrics were compared to the preceding instance for each user (e.g., the last screening prior to the 4-month COVID-19 stay-at-home mandate), using statistical methods (e.g., Student's $t$-test). Average temporal metrics for the subset of individuals $(n=8)$ are shown in Fig. 4. The average time to complete functional tasks increased after the COVID-19 stay-at-home mandates and interventional program restrictions for all functional areas (e.g., motor, memory, speech, executive function, and dual-tasks), taking about 2.1 times longer. Tasks denoted with an asterisk $(*)$ showed a significant increase $(p<0.05)$ in time from before the COVID-19 restrictions to after. Further, accuracy metrics (e.g., distance from true value points) for fine motor, executive function, and dualtask assessments also show degradation due to the COVID-19 restrictions (Fig. 5). Overall average distances increased by a factor of 1.55 , and in timed assessments the number of correct responses and screen interactions for users decreased following the stay-at-home mandate by a factor of 1.43 (Fig. 5). Tasks denoted with an asterisk $(*)$ in Fig. 5 indicate a significant increase $(p<0.05)$ in distances drawn or moved for fine and gross dual-tasks and a significant decrease $(p<0.05)$ in the accuracy count for a reflex test. 


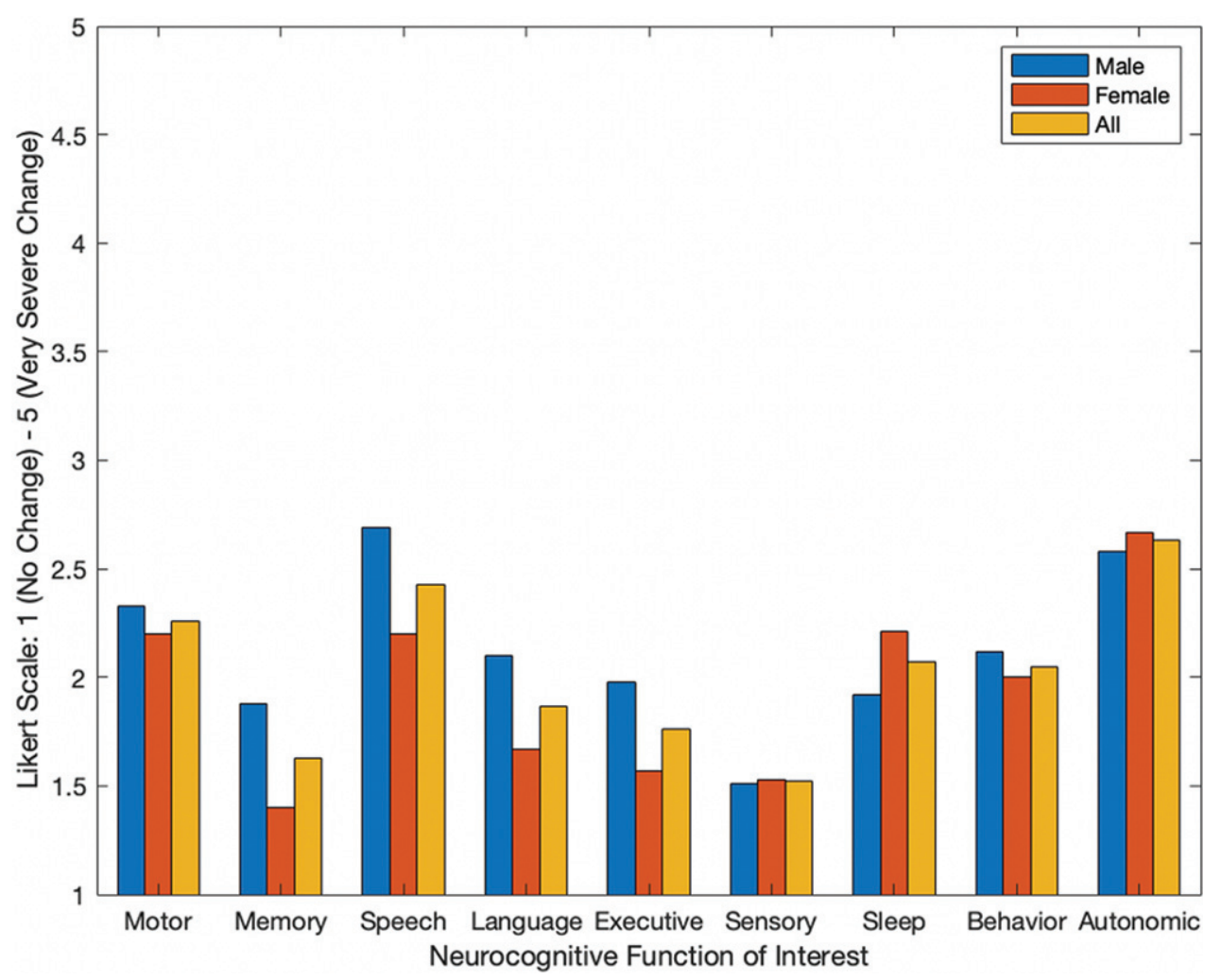

Fig. 3. Average self-reporting of changes in symptoms after the COVID-19 stay-at-home mandate for individuals with PD across all neurocognitive categories.

\section{DISCUSSION}

\section{Self-reporting}

Although individuals reported various timing factors for when they felt at their best, the highest reported instance for the population was after exercise $(67.86 \%)$. Additional insights from collected questionnaires showed that individuals maintained, on average, the number of days in which they were active following the restrictions imposed by COVID19 ( $p>0.05$ for all groups). However, the number of active minutes per day following these restrictions was significantly less compared to the period before the stay-at-home mandate $(p=0.022)$. The number of different activities completed by the population was also reduced due to the COVID-19 restrictions (Table 1). Although the majority of individuals felt at their best after exercising, the changes in activity levels as well as the type of physical activity (e.g., the change from structured physical intervention protocols to self-guided activity) during this period of time prompted a focus on understanding the individuals' symptom progression due to decreased activity.
While individuals with PD may manifest different symptoms and at different rates, the overall highest reported changes of symptoms were autonomic function, speech, motor, and sleep (Fig. 3). Primary manifestations of PD include abnormalities of movement (e.g., akinesia, rigidity, and tremor) and variably present manifestations (e.g., dysarthria, difficulty performing simultaneous actions, and fatigue) [6]. Any changes in both primary and/or variably present manifestations would subsequently link to self-reported changes in functions like motor and speech. Further, individuals reported symptoms across all neurocognitive areas of interest (e.g., motor, memory, speech, language, executive function, sensory, behavioral and psychological state, sleep, and autonomic function). This can be seen in Fig. 3 as, on average, all values are higher than 1 (e.g., which denotes 'no change' on the administered Likert scale).

Of the self-reporting individuals, $82.14 \%$ indicated having at least one symptom that worsened moderately or higher on the Likert scale (e.g., having one symptom with a Likert value of at least 3 ), and $67.86 \%$ or individuals reported multiple symptoms 


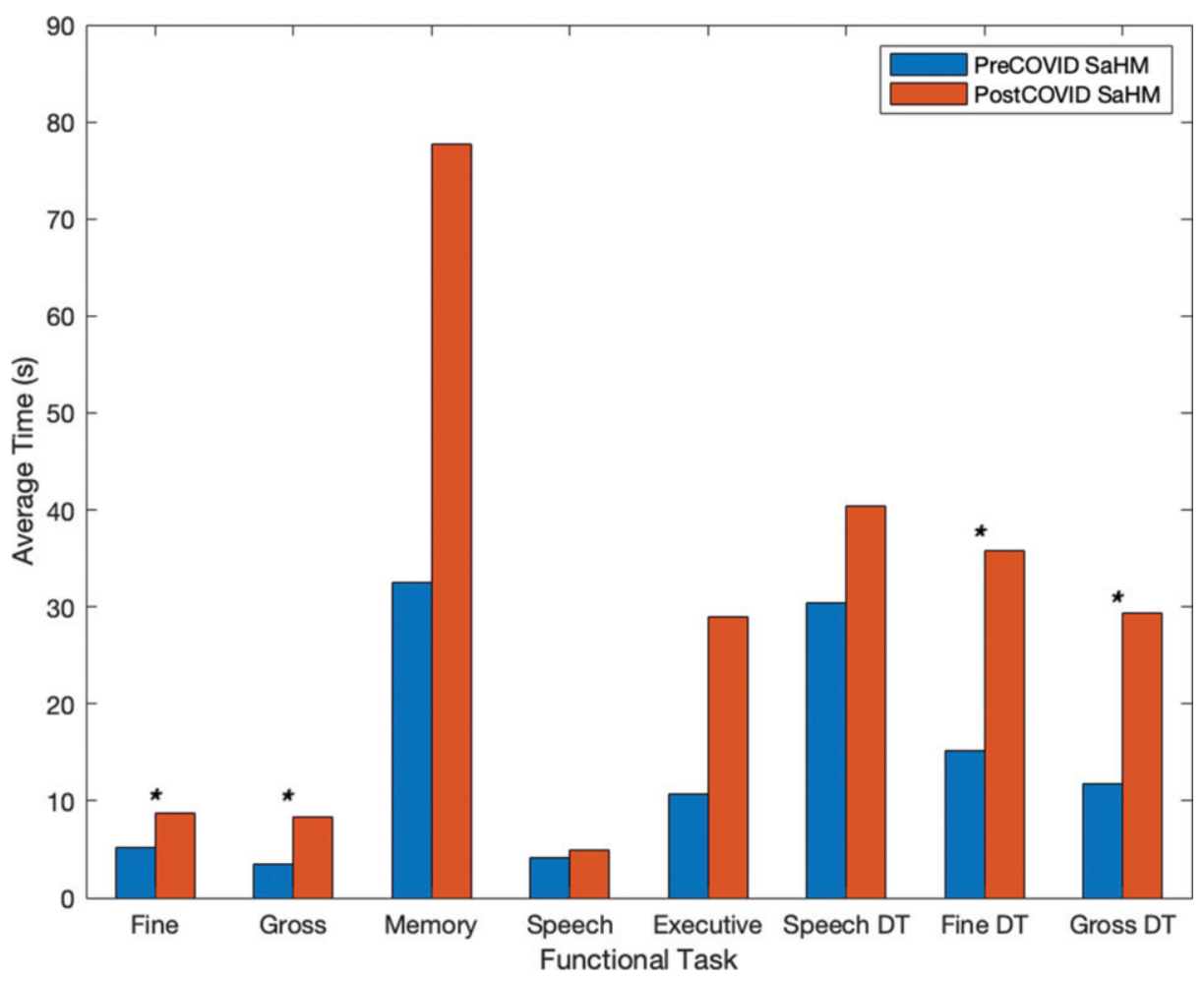

Fig. 4. Individuals' timing metrics prior to and following the COVID-19 stay-at-home mandate.

that worsened moderately or higher during the time of the COVID-19 stay-at-home mandates and in-person intervention program restrictions. Further, over half of the self-reporting individuals $(57.14 \%)$ reported having at least one symptom worsen severely or very severely (e.g., one symptom with a Likert value of at least 4 ) and $42.86 \%$ indicated that they had multiple symptoms change severely or very severely.

Not only is physical activity beneficial for individuals with $\mathrm{PD}$, maintaining those structured interventions in a socially engaging and comfortable setting is also necessary for minimizing the effects of disease progression $[4,12,15]$.

Information collected from the self-reporting questionnaire showed that the second most important factor to individuals with PD in feeling their best was being in a comfortable and supportive environment $(60.71 \%)$. The majority of physical rehabilitation programs that individuals with PD interact in are conducted outside the home in structured and supervised group settings. These in-person physical intervention programs not only provide tailored exercise protocols but also create a supportive environment that encourages social engagement among participants. The inherent nature of structured exercise programs allows for the inclusion of two highly ranked factors for individuals diagnosed with PD to feel their best (e.g., after exercise, and being in a comfortable and supportive environment).

\section{Mobile application testing}

A further analysis of quantitative mobile assessments was also completed to give a deeper understanding of how individuals symptoms were affected by the COVID-19 stay-at-home mandates and inperson intervention program restrictions. This analysis was done by comparing collected functional task results from an individual's baseline assessment (e.g., prior to the COVID-19 stay-at-home mandate) to an assessment taken following their return to programs.

Individuals showed longer completion times on average for each of the functional tests (e.g., motor, memory, speech, executive function, and dual-task assessments) as seen in Fig. 4. Overall results showed that timing metrics for the group worsened on average (e.g., taking 2.1 times longer across functional tasks). Timing for fine motor, gross motor, and motor dual-task assessments showed a significant decline following the COVID-19 restrictions $(p<0.034)$ as denoted by an asterisk $\left({ }^{*}\right)$ in the figure. Similarly, individuals showed, on average, decreased accuracy. 


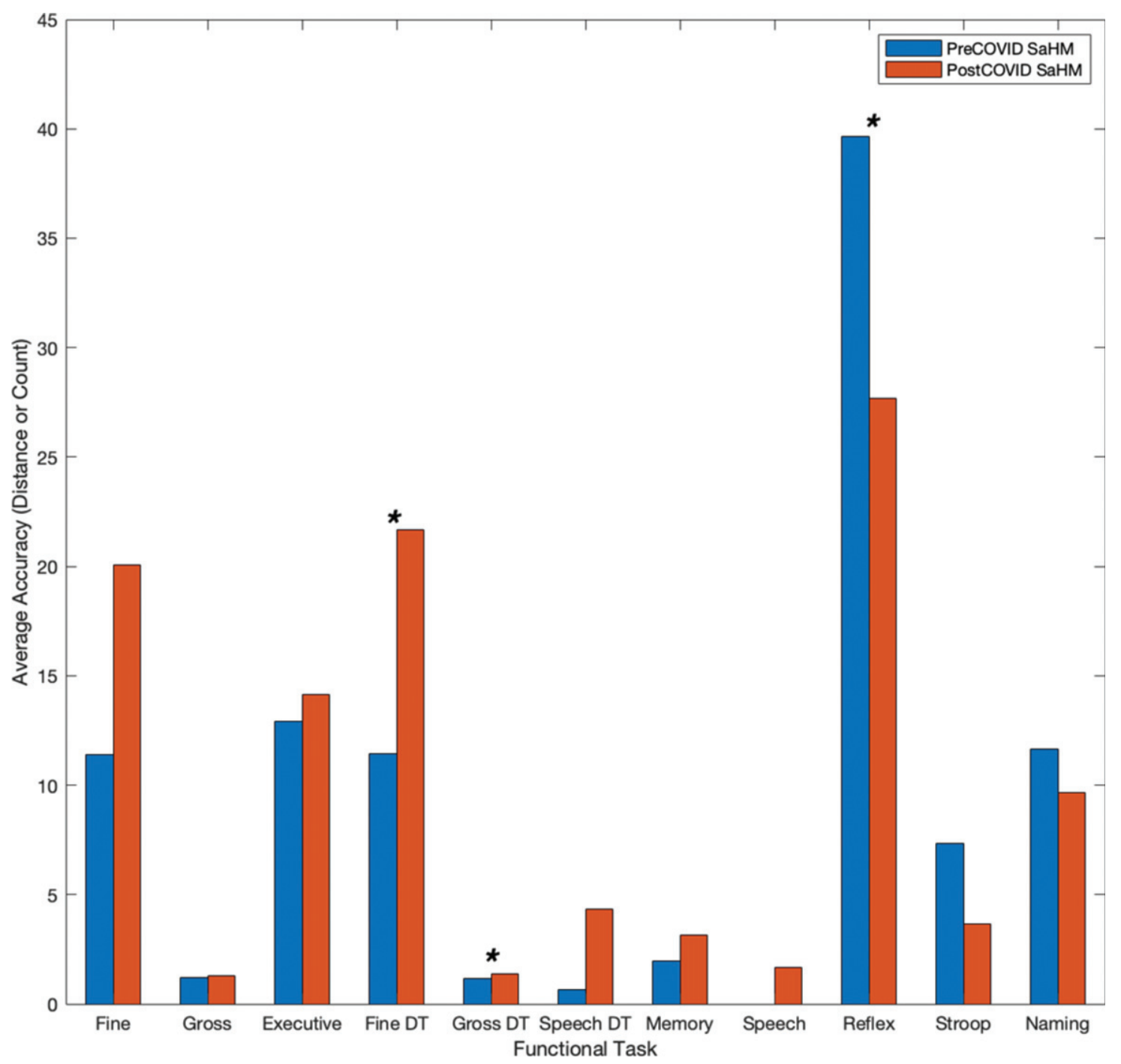

Fig. 5. Individuals' accuracy metrics prior to and following the COVID-19 stay-at-home mandate.

In untimed tasks (e.g., fine motor tracing, trail making tasks, and dual-task assessments) average distance from true value points increased as did count measures for speech errors and card matching following the COVID-19 restrictions. In timed tasks (e.g., reflex, SWCT, and object naming) count measures decreased following the COVID-19 restrictions (Fig. 5). Average distance metrics for dual-task fine motor and gross motor function showed a significant decline following the stay-at-home mandate $(p<0.044)$ as did the average count measure for a reflex-based test $(p=0.010)$ as indicated by an asterisk $(*)$.

The testing completed using a mobile-based assessment allowed for both the comparison and confirmation of self-reported symptoms from participants. Motor function and speech were two of the highest reported symptoms that changed due to the COVID-19 stay-at-home mandates and in-person intervention program restrictions. A quantitative analysis showed there was a significant difference with respect to time for task completion of functional tasks involving both of these functions. Motor tasks (e.g., tracing shapes on the device screen and manipulating the device in space) showed significant declines in time $(p<0.037)$ following the COVID-19 restrictions. Similarly, dual-task assessments, which prompted the user to complete a non-automatic speech task (e.g., listing the months of the year, aloud, in reverse order; December to January) in tandem 
with a motor task (e.g., tracing a variety of shapes on the device screen or manipulating the device in space), showed significant differences with respect to the time for task completion $(p<0.007)$.

This research was completed in tandem for concurrent projects on mobile based neurocognitive assessments for individuals with PD. A major limitation of this work was the limited sample of participants. This limitation arose as access to the intended population, through structured intervention programs, was discontinued due to stay-at-home mandates. Limitations continued following stay-athome mandates with additional restrictions set by state departments and individuals maintaining distancing protocols as they are notably at higher risk due to comorbidities. Further, all participants in this study participated in an exercise program prior to the stay-at-home mandates which presents a sampling bias. Individuals with PD who do not participate in any structured exercise programs were not represented, therefore, the following conclusions cannot be generalized to the entire population of individuals with PD, but rather those who participated in structured physical activities as a means of recommended adjunct interventions. Further work should be completed to understand how all diagnosed individuals' physical and mental health are affected by stay-at-home orders. Future work should also be completed to see how individuals' symptoms progress following the re-initiation of structured rehabilitative interventions. Finally, the continued development and implementation of mobile-based health monitoring technology should be prioritized to allow for a higher quality of user assessment and care in the event of similar circumstances.

\section{CONCLUSIONS}

The COVID-19 stay-at-home mandates and restrictions were intended to help minimize individuals, especially those at high risk, from coming into contact with the virus. However, these mandates also removed many individuals from participating in valuable, structured, in-person physical intervention programs along with natural support systems created by these group settings. For those with PD, both physical activity and being in comfortable and supportive environments are essential to feeling at their best. The self-reporting of individuals and statistical analyses show that the inability to participate in in-person intervention programs had direct negative effects on their activity levels and overall symptom progression, both of which directly affect the individual's quality of life; however, an emphasis needs to be placed on future work and a larger population. As scientific advances are made for the possibility of future global pandemics, a strong focus should be placed on maintaining these recommended and beneficial programs. Telemedicine based approaches for these intervention therapies, could allow for the continued participation and rehabilitation of diagnosed individuals in both a safe and supportive setting. Further, expanding mobile-based technologies for the purpose of collecting and assessing additional objective metrics for all areas of neurocognition is necessary in the confirmation of all self-reported symptoms.

\section{CONFLICT OF INTEREST}

All authors of this work declare that there are no conflicts of interest in the authorship or publication of this contribution.

\section{SUPPLEMENTARY MATERIAL}

The supplementary material is available in the electronic version of this article: https://dx.doi.org/ 10.3233/JPD-212553.

\section{REFERENCES}

[1] Rodriguez M, Rodriguez-Sabate C, Morales I, Sanchez A, Sabate M (2015) Parkinson's disease as a result of aging. Aging Cell 14, 293-308.

[2] Collier TJ, Kanaan NM, Kordower JH (2017) Aging and Parkinson's disease: Different sides of the same coin? Mov Disord 32, 983-990.

[3] Zlokovic BV (2011) Neurovascular pathways to neurodegeneration in Alzheimer's disease and other disorders. Nat Rev Neurosci 12, 723-738.

[4] Borrione P, Tranchita E, Sansone P, Parisi A (2014) Effects of physical activity in Parkinson's disease: A new tool for rehabilitation. World J Methodol 4, 133-143.

[5] Bloem BR, Hausdorff JM, Visser JE, Giladi N (2004) Falls and freezing of gait in Parkinson's disease: A review of two interconnected, episodic phenomena. Mov Disord 19, 871-884.

[6] Mazzoni P, Shabbott B, Cortés JC (2012) Motor control abnormalities in Parkinson's disease. Cold Spring Harbor Persp Med 2, a009282.

[7] Yang Y, Tang BS, Guo JF (2016) Parkinson's disease and cognitive impairment. Parkinsons Dis 2016, 6734678.

[8] Barbosa AF, Voos MC, Chen J, Francato DCV, Souza CO, Barbosa ER, Chien HF, Mansur LL (2017) Cognitive or cognitive-motor executive function tasks? Evaluating verbal fluency measures in people with Parkinson's disease. Biomed Res Int 2017, 7893975. 
[9] Templeton JM, Poellabauer C, Schneider S (2020) Enhancement of neurocognitive assessments using smartphone capabilities: Systematic review. JMIR Mhealth Uhealth $\mathbf{8}$, e15517.

[10] Blake-Krebs B, Herman L (2001) When Parkinson's strikes early: Voices, choices, resources, and treatment. Hunter House.

[11] Vaartio-Rajalin H, Rauhala A, Fagerström L (2019) Person-centered home-based rehabilitation for persons with Parkinson's disease: A scoping review. Int J Nurs Stud 99, 103395.

[12] Lauzé M, Daneault J F, Duval C (2016) The effects of physical activity in Parkinson's disease: A review. J Parkinsons Dis 6, 685-698.

[13] Schenkman M, Hall DA, Barón AE, Schwartz RS, Mettler P, Kohrt WM (2012) Exercise for people in early- or mid-stage Parkinson disease: A 16-month randomized controlled trial. Phys Ther 92, 1395-1410.

[14] Brooke J, Jackson D (2020) Older people and COVID-19: Isolation, risk and ageism. J Clin Nurs 29, 2044-2046.

[15] Zaman A, Ellingson L, Sunken A, Gibson E, Stegemöller EL (2021) Determinants of exercise behaviour in persons with Parkinson's disease. Disabil Rehabil 43, 696-702.

[16] Sajatovic M, Ridgel AL, Walter EM, Tatsuoka CM, ColónZimmermann K, Ramsey RK, Welter E, Gunzler SA, Whitney CM, Walter BL (2017) A randomized trial of individual versus group-format exercise and self-management in individuals with Parkinson's disease and comorbid depression. Patient Prefer Adherence 11, 965-973.

[17] Hindle JV, Hurt CS, Burn DJ, Brown RG, Samuel M, Wilson KC, Clare L (2016) The effects of cognitive reserve and lifestyle on cognition and dementia in Parkinson's disease-a longitudinal cohort study. Int J Geriatr Psychiatry 31, 13-23.

[18] Sampson EL, Bulpitt CJ, Fletcher AE (2009) Survival of community-dwelling older people: The effect of cognitive impairment and social engagement. J Am Geriatr Soc 57, 985-991.

[19] Pfefferbaum B, North CS (2020) Mental health and the Covid-19 pandemic. N Engl J Med 383, 510-512.

[20] Vega J, Couth S, Poliakoff E, Kotz S, Sullivan M, Jay C, Harper S (2018) Back to analogue: Self-reporting for
Parkinson's disease. In Proceedings of the 2018 CHI conference on human factors in computing systems, pp. 1-13.

[21] Amara AW, Chahine L, Seedorff N, Caspell-Garcia CJ, Coffey C, Simuni T, Parkinson's Progression Markers Initiative (2019) Self-reported physical activity levels and clinical progression in early Parkinson's disease. Parkinsonism Relat Disord 61, 118-125.

[22] Mischley LK, Lau RC, Weiss NS (2017) Use of a self-rating scale of the nature and severity of symptoms in Parkinson's disease (PRO-PD): Correlation with quality of life and existing scales of disease severity. NPJ Parkinsons Dis 3, 20.

[23] Nasreddine ZS, Phillips NA, Bédirian V, Charbonneau S, Whitehead V, Collin I, Cummings JL, Chertkow H (2005) The Montreal Cognitive Assessment, MoCA: A brief screening tool for mild cognitive impairment. J Am Geriatr Soc 53, 695-699.

[24] Tombaugh TN, McIntyre NJ (1992) The Mini-Mental State Examination: A comprehensive review. J Am Geriatr Soc 40, 922-935.

[25] Al-Heizan MO, Giles GM, Wolf TJ, Edwards DF (2020). The construct validity of a new screening measure of functional cognitive ability: The menu task. Neuropsychol Rehabil 30, 961-972.

[26] MacNeill Horton A (2008) The Neuropsychology Handbook Third Edition. Springer Publishing Company.

[27] Czuba KJ, Kersten P, Kayes NM, Smith GA, Barker-Collo S, Taylor WJ, McPherson KM (2016) Measuring neurobehavioral functioning in people with traumatic brain injury: Rasch analysis of neurobehavioral functioning inventory. $J$ Head Trauma Rehabil 31, E59.

[28] Post L, Van Den Heuvel T, Van Prooije X, Van Ruissen B, Van De Warrenburg B, Nonnekes J (2020) Young onset Parkinson's disease: A modern and tailored approach. $J$ Parkinsons Dis 10(s1), S29-S36.

[29] Templeton JM, Poellabauer C, Schneider S (2021) Design of a mobile-based neurological assessment tool for aging populations. In Wireless Mobile Communication and Healthcare, Ye J, O'Grady MJ, Civitarese G, Yordanova K, eds. MobiHealth 2020. Lecture Notes of the Institute for Computer Sciences, Social Informatics and Telecommunications Engineering, vol 362. Springer, Cham. 